ARTICLE

https://doi.org/10.1038/s41467-018-08215-5

\title{
Quantized angular momentum in topological optical systems
}

\author{
Mário G. Silveirinha (1) ${ }^{1}$
}

The Chern index characterizes the topological phases of nonreciprocal photonic systems. Unlike in electronics, the photonic Chern number has no clear physical meaning, except that it determines the number of unidirectional edge states supported by an interface with a trivial mirror. Here, we fill in this gap by demonstrating that the photonic Chern number can be understood as the quantum of the light-angular momentum in a photonic insulator cavity. It is proven that for a large cavity, the thermal fluctuation-induced angular momentum is precisely quantized in the band-gaps of the bulk states. The nontrivial expectation of the light angular momentum is due to a circulation of thermal energy in closed orbits. Remarkably, this result can be extended to systems without a topological classification, and in such a case the "quantum" of the angular momentum density is determined by the net number of unidirectional edge states supported by the cavity walls.

\footnotetext{
${ }^{1}$ University of Lisbon-Instituto Superior Técnico and Instituto de Telecomunicações, Avenida Rovisco Pais, 1, 1049-001 Lisboa, Portugal. Correspondence and requests for materials should be addressed to M.G.S. (email: mario.silveirinha@co.it.pt)
} 
$\mathrm{T}$ opological matter and topological effects have elicited a great deal of interest in recent years, first in electronics ${ }^{1-5}$ and then in photonics and acoustics ${ }^{6-13}$. Notably, topological materials may enable the propagation of waves totally immune to the back-scattering due to either deformations of the propagation path, sharp corners, or due to defects. Hence, topological systems may enable a more efficient transport of light and avoid unwanted feedback and loss due to backreflections $s^{6-13}$. There are several sub-classes of photonic materials with topological properties ${ }^{9-11}$, but only optical systems with a broken time-reversal symmetry provide a strong topological protection ${ }^{14,15}$. In such systems, the different topological phases are characterized by a topological index known as the Chern number.

In the fermionic case the Chern number has an immediate physical meaning: it determines the value of the quantized Hall conductivity of a $2 \mathrm{D}$ electron gas ${ }^{4,16}$, and thus the electronic transport properties in the zero temperature limit. In contrast, in photonics the Chern number has not been linked with any specific physical quantity, except that similar to electronics it determines the number of gapless unidirectional edge states at an interface with a trivial insulator ${ }^{6,8,14,15}$. In this article, we fill in this gap of understanding by proving that the (thermal or quantum) fluctuation-induced light angular momentum in a generic photonic-insulator cavity in thermodynamic equilibrium with a large reservoir has a spectral density per unit of area that is quantized in units of $\frac{1}{\pi c^{c}} \mathcal{E}_{T, \omega}$, with $\mathcal{E}_{T, \omega}=\frac{\hbar \omega}{2} \operatorname{coth}\left(\frac{\hbar \omega}{2 k_{\mathrm{B}} T}\right)$ the mean energy of a harmonic oscillator at temperature $T^{17}$. The nontrivial light angular momentum is due to a circulation of "heat" in closed orbits ${ }^{18}$. This result is rather general and remarkably applies even to systems without any topological classification. In the case of topological systems, the quantized angular momentum spectral density is precisely determined by the photonic Chern number.

\section{Results}

The topological cavity. We consider a generic closed cavity filled with either a photonic crystal or, alternatively, an electromagnetic continuum with no intrinsic periodicity (Fig. 1). The cavity crosssection shape in the $x o y$-plane can be rather arbitrary, but for simplicity, we focus on a rectangular cross-section with

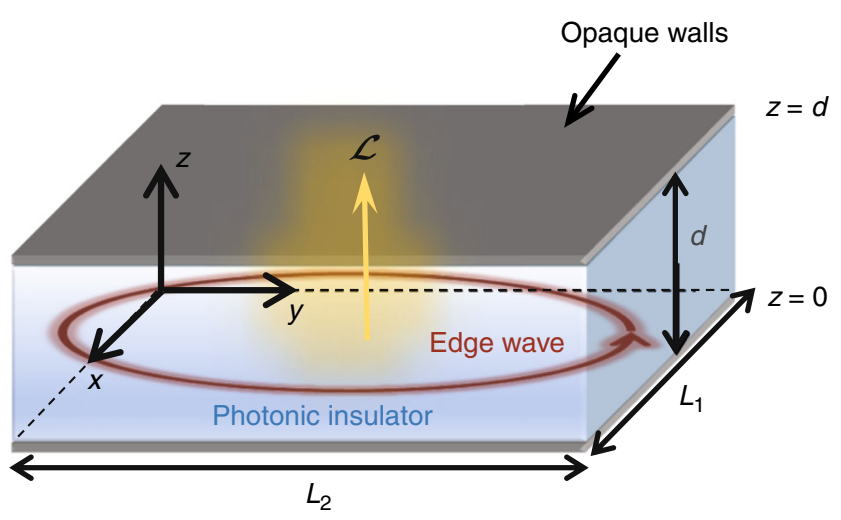

Fig. 1 Geometry of a generic "photonic insulator" cavity. The closed electromagnetic cavity does not support bulk states in some spectral range ("photonic insulator"). The lateral walls (not shown) may eventually enable the propagation of edge-type waves confined to the boundary. The thermal fluctuation-induced light is generally characterized by a non-trivial angular momentum $\mathcal{L}$, which for a sufficiently large cavity has a quantized spectral density in a band-gap dimensions $L_{1} \times L_{2}$. The cavity's height along the $z$-direction is $d$. The cavity walls are assumed "opaque", i.e., impenetrable by light. For example, perfectly electric conducting (PEC) walls are opaque. The cavity may be regarded as a parallel-plate waveguide (with propagation plane parallel to $x o y$ ) terminated with the lateral walls. The equivalent unbounded waveguide does not support electromagnetic states in the spectral range of interest (photonic insulator). Note that the band structure is determined not only by the materials inside the cavity but also by the top and bottom walls. For simplicity, the effects of material loss in the wave propagation are assumed negligible. Recently, it was shown that topological concepts may be extended to some nonHermitian systems, e.g., systems with loss and or gain ${ }^{19-23}$.

Importantly, even when there are no bulk states, the opaque boundaries may enable the propagation of edge states localized at the cavity lateral walls 18,24 . Such edge states famously occur in topological platforms $s^{6,7,9,14,15}$, but they may as well emerge in systems with no topological classification ${ }^{25}$. Furthermore, the edge-states may be supported even when the system is timereversal invariant (reciprocal) $)^{9,24,25}$.

Angular momentum of an edge state. Consider a generic edgestate circulating around the cavity lateral walls (Fig. 1). The circulating motion is evidently associated with a non-trivial light angular momentum given by $\mathcal{L}=\frac{1}{c^{2}} \int \mathrm{d} V \mathbf{r} \times \mathbf{S}$, where $\mathbf{S}$ is the Poynting vector, $\mathbf{r}=(x, y, z)$ is a generic point in space, and the integration is over the cavity volume. The angular momentum of photonic systems is extensively discussed in refs. ${ }^{26-29}$. With reference to the nomenclature of ref. ${ }^{29}$, we adopt a "kinetic picture" and the Abraham formalism so that the electromagnetic momentum density is $\mathbf{S} / c^{2}{ }^{30-32}$. In general, the angular momentum may be decomposed into orbital and spin components ${ }^{29}$. The spin component is origin independent, but for an open system the orbital component (and thereby also the total angular momentum) generally depends on the origin of the coordinate $\operatorname{axes}^{29}$. In contrast, for a closed cavity it can be shown that the total Abraham light momentum $\left(\int \mathrm{d} V \mathbf{S} / c^{2}\right)$ of a mode always vanishes, and thereby the angular momentum $\mathcal{L}$ is origin independent.

In the Supplementary Note 1, it is shown that for a sufficiently large cavity $\left(d \ll L_{i}, i=1,2\right)$ the $z$-component of the angular momentum is

$$
\frac{\mathcal{L}_{z}}{A_{\text {tot }}} \approx s \frac{2}{c^{2} l_{\mathrm{P}}}\left|\int_{\text {cav. perimeter }} \mathrm{d} x_{\|} \int \mathrm{d} x_{\perp} \int \mathrm{d} z S_{\|}\right|,
$$

where $A_{\text {tot }}=L_{1} \times L_{2}$ is the cross-sectional area, $l_{\mathrm{p}}=2\left(L_{1}+L_{2}\right)$ is the cavity perimeter, and $s=+1(s=-1)$ for a mode that circulates in the anti-clockwise (clockwise) direction, respectively. The coordinate $x_{\|}$is measured along the cavity perimeter and the coordinate $x_{\perp}$ along the perpendicular direction $\left(x_{\|}\right.$and $x_{\perp}$ are in the xoy plane). Furthermore, $S_{\|}$is the Poynting vector component parallel to the cavity walls. Similar to the theory of photonic crystals $^{33}$, the spatially averaged Poynting vector along the propagation path can be related to the (net) group velocity $v_{\mathrm{g}}$ of the edge-wave and with the energy stored in the cavity $\mathcal{E}$ as follows: $\int_{\text {cav. perimeter }} \mathrm{d} x_{\|} \int \mathrm{d} x_{\perp} \int \mathrm{d} z S_{\|}=v_{\mathrm{g}} \mathcal{E}$ (see the Supplementary Note 2 and the Supplementary Figure 1). Hence, the angular momentum can be written as

$$
\frac{\mathcal{L}_{z}}{A_{\text {tot }}} \approx s \frac{2}{c^{2} l_{\mathrm{P}}}\left|v_{\mathrm{g}}\right| \mathcal{E}
$$


The correction term that makes the two sides of the equation identical vanishes in the limit $A_{\text {tot }} \rightarrow \infty$. In practice, the equation holds true when the cavity diameter is a few times larger than the characteristic modal size of the edge wave.

Angular momentum of thermal light. Suppose now that the system is in thermodynamic equilibrium with a reservoir at temperature $T$, and that all the light inside the cavity is generated by thermal (or quantum) fluctuations. Crucially, a few recent works $18,32,34,35$ have shown that a thermodynamic equilibrium (without any heat sources) is compatible with a circulation of thermal energy in closed orbits. Specifically, for nonreciprocal systems the expectation of the heat current ${ }^{18,35}$ and of the angular momentum ${ }^{18}$ can be nontrivial. In the limit of vanishingly small material loss, the angular momentum expectation can be written as $\left\langle\mathcal{L}_{z}\right\rangle=\sum_{\omega_{n}>0} \mathcal{E}_{T, \omega_{n}} \mathcal{L}^{(n)}$ where the summation is over all the positive frequency modes of the cavity. Here, $\mathcal{L}^{(n)}$ is the angular momentum of a generic mode normalized to its energy and $\omega_{n}$ is the oscillation frequency. The formula of $\left\langle\mathcal{L}_{z}\right\rangle$ is consistent with the fluctuation-dissipation theorem (see ref. ${ }^{18}$ ). The (unilateral) angular momentum spectral density $\mathcal{L}_{\omega}$ is defined such that $\left\langle\mathcal{L}_{z}\right\rangle=\int_{0}^{\infty} d \omega \mathcal{L}_{\omega}$. It has the explicit expression $\mathcal{L}_{\omega}=\mathcal{E}_{T, \omega} \sum_{\omega_{n}>0} \mathcal{L}^{(n)} \delta\left(\omega-\omega_{n}\right)$. The angular momentum expectation may be nontrivial only when the time-reversal symmetry is broken, because otherwise the heat current vanishes $\left(\left\langle\mathbf{S} / c^{2}\right\rangle=0\right)$ in all points of the cavity ${ }^{18,35}$.

In a band-gap all the cavity modes must be edge-waves. Using Eq. (2) to evaluate the angular momentum $\left(\mathcal{L}^{(n)}\right)$ of a generic edge-state it is found that:

$$
\frac{\mathcal{L}_{\omega}}{A_{\mathrm{tot}}} \approx \mathcal{E}_{T, \omega} \frac{2}{c^{2} l_{\mathrm{P}}} \sum_{n} s_{n}\left|v_{\mathrm{g}, n}\right| \delta\left(\omega-\omega_{n}\right), \quad \text { (in a band-gap). }
$$

The summation is over all the (positive-frequency) edge modes in the spectral range of interest. Here, $v_{\mathrm{g} . n}$ is the group velocity of the $n$th mode and $s_{n}= \pm 1$ depending if the energy flows in the anti-clockwise or clockwise direction. As discussed in the Supplementary Note 2, the edge modes can be organized in branches $(m=1,2, \ldots)$, and the dispersion of each branch is of the form $\omega_{m}\left(k_{\|}\right)$. The parameter $k_{\|}$determines the total phase delay $\left(k_{\|} l_{\mathrm{P}}\right)$ acquired by the wave as it travels one complete loop around the cavity. Even though $k_{\|}$may be regarded as a continuous variable, only solutions with $k_{\|} l_{\mathrm{P}}=2 \pi n$ with $n$ integer are physical. Thus, for a large cavity perimeter and for a given branch the sum over the edge-waves can be approximated by an integral: $\frac{1}{l_{\mathrm{p}}} \sum_{n} \rightarrow \frac{1}{2 \pi} \int \mathrm{d} k_{\|}$. This shows that $\frac{\mathcal{L}_{\omega}}{A_{\mathrm{tot}}} \approx \mathcal{E}_{T, \omega} \frac{1}{c^{2} \pi} \sum_{m} \int \mathrm{d} k_{\|} s_{m}\left|v_{\mathrm{g}, m}\right| \delta\left(\omega-\omega_{m}\right)$. But since $v_{\mathrm{g}, m}=\frac{\partial \omega_{m}}{\partial k_{\|}}$ (see the Supplementary Note 2), we finally conclude that:

$$
\frac{\mathcal{L}_{\omega}}{A_{\text {tot }}} \approx-\mathcal{E}_{T, \omega} \frac{1}{c^{2} \pi} \mathcal{C}_{\omega}, \quad \text { with } \quad \mathcal{C}_{\omega}=-\sum_{\omega_{m}=\omega} s_{m} .
$$

The sum is over all the edge-modes for which $\omega_{m}=\omega$ (each branch may contribute with one or more points). Since $s_{m}= \pm 1$ the parameter $\mathcal{C}_{\omega}$ simply counts the difference between the number of edge modes associated with an anti-clockwise power flow $\left(s_{m}=+1\right)$ and the number of edge modes associated with a clockwise power flow $\left(s_{m}=-1\right)$ at frequency $\omega$. In particular, we see that the absolute value of the sum gives the net number of unidirectional modes. This is the first key result of the article. It establishes that in the band-gaps of a photonic insulator cavity, the expectation of the (Abraham) angular momentum spectral density per unit of area is quantized in units of $\frac{1}{\pi c^{2}} \mathcal{E}_{T, \omega}$. The quantization is strictly valid in the continuum limit, $A_{\text {tot }} \rightarrow \infty$ (likewise, in electronic systems the Hall conductivity is quantized only when the sample area approaches infinity). The quantized angular momentum density is determined by the net number of unidirectional edge modes $\left(\mathcal{C}_{\omega}=0, \pm 1, \pm 2, \ldots\right)$ at frequency $\omega$. This property is rather general and does not depend on the topological nature of the system. Defects may lead to additional contributions to the angular momentum, but their presence can be safely neglected in state-of-the-art photonic designs. Furthermore, it is shown in the Supplementary Note 3 that the topological cavity may be regarded as a circular transmission line and that the power spectral density associated with the energy flow around the cavity walls is also quantized.

The angular momentum quantum. Let us now focus on topological Chern-type materials ${ }^{14,15,36,37}$. The bulk-edge correspondence principle establishes that the net number of unidirectional edge modes at the interface of two topological materials is coincident with the gap Chern number difference ${ }^{6,37}$. Thus, supposing that the cavity walls are topologically trivial, (e.g., PEC walls) it follows that $\left|\mathcal{C}_{\omega}\right|=\left|\mathcal{C}_{\text {gap }}\right|$, where $\mathcal{C}_{\text {gap }}$ is the gap Chern number of the photonic insulator. This is the second key result of the article. It implies that the photonic Chern number has a precise physical meaning: it determines the quantized angular momentum spectral density of a cavity in a thermodynamic equilibrium. In particular, we see that the fluctuation-induced angular momentum spectral density is totally insensitive to any variation of the system parameters (e.g., a change of the biasing magnetic field) that does not close the gap. Note that the Chern number can be unambiguously defined for fully $3 \mathrm{D}$ waveguide platforms ${ }^{38,39}$. Furthermore, a more sophisticated analysis reported elsewhere demonstrates that one has precisely $\mathcal{C}_{\omega}=\mathcal{C}_{\text {gap }}{ }^{40}$ (the numerical examples presented in the following confirm this result; here, $\mathcal{C}_{\text {gap }}$ is the sum of the Chern numbers of the bands below the gap, including negative frequency bands. The Chern number of a given band is defined consistently with refs. $36,38: \mathcal{C}_{n}=\hat{\mathbf{z}}$. $\nabla_{\mathbf{k}} \times \mathcal{A}_{n \mathbf{k}}$ with the Berry potential given by $\left.\mathcal{A}_{n \mathbf{k}}=i\left\langle\mathbf{Q}_{n \mathbf{k}} \mid \partial_{\mathbf{k}} \mathbf{Q}_{n \mathbf{k}}\right\rangle\right)$. Thus, a positive (negative) gap Chern number implies that the unidirectional edge waves propagate clockwise (anti-clockwise) with respect to the $z$-axis.

Magnetized electric plasma cavity. To illustrate the application of the developed ideas, we take the photonic insulator as a magnetized electric plasma with a gyrotropic permittivity response, $\bar{\varepsilon}=\varepsilon_{\mathrm{t}} \mathbf{1}_{\mathrm{t}}+\varepsilon_{\mathrm{a}} \hat{\mathbf{z}} \otimes \hat{\mathbf{z}}+i \varepsilon_{\mathrm{g}} \hat{\mathbf{z}} \times 1$, with $\mathbf{1}_{\mathrm{t}}=\hat{\mathbf{x}} \otimes \hat{\mathbf{x}}+\hat{\mathbf{y}} \otimes \hat{\mathbf{y}}$ and

$$
\begin{array}{r}
\varepsilon_{\mathrm{t}}=1-\frac{\omega_{\mathrm{p}}^{2}(1+i \gamma / \omega)}{(\omega+i \gamma)^{2}-\omega_{\mathrm{c}}^{2}}, \varepsilon_{\mathrm{a}}=1-\frac{\omega_{\mathrm{p}}^{2}}{\omega(\omega+i \gamma)}, \text { and } \\
\varepsilon_{\mathrm{g}}=\frac{1}{\omega} \frac{\omega_{\mathrm{c}} \omega_{\mathrm{p}}^{2}}{\omega_{\mathrm{c}}^{2}-(\omega+i \gamma)^{2}}
\end{array}
$$

where $\omega_{\mathrm{p}}$ is the plasma frequency, $\gamma$ is the collision frequency, $\omega_{c}=-q B_{0} / m$ is the cyclotron frequency $\left(\omega_{c}>0\right.$ when the magnetic field is directed along $+z), q=-e$ is the electron charge and $m$ is the electron effective mass ${ }^{41}$. The bias magnetic field is $\mathbf{B}=B_{0} \hat{\mathbf{z}}$. InSb with a magnetic bias and other narrow-gap semiconductors have a similar gyrotropic response at terahertz frequencies ${ }^{42,43}$. 


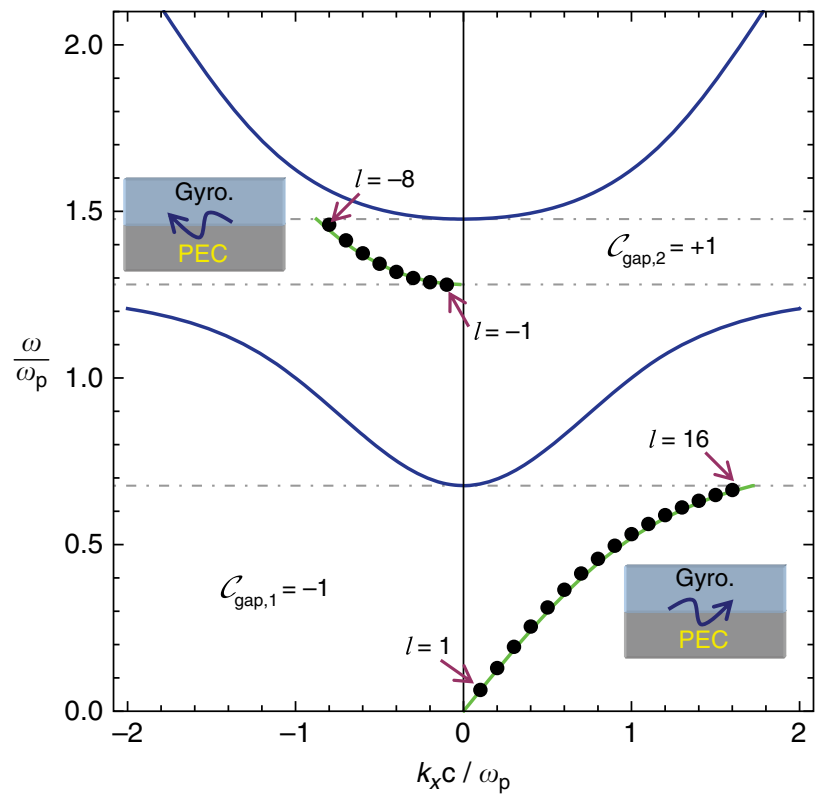

Fig. 2 Dispersion of the bulk modes and of the edge waves. Blue lines: band diagram of the bulk gyrotropic material with $\omega_{\mathrm{c}}=0.8 \omega_{\mathrm{p}}$. The two photonic band-gaps are delimited by the dashed horizontal grid lines. The gap Chern numbers $\left(\mathcal{C}_{\text {gap }, 1}\right.$ and $\left.\mathcal{C}_{\text {gap }, 2}\right)$ are given in the insets. Green lines: dispersion of the (gap) edge modes supported by a planar interface $(y=0)$ between the gyrotropic material (region $y>0$ ) and PEC material (region $y<0$ ). The edge modes propagate along the $x$-axis (see the insets). The edge mode dispersion is shown only in the band-gaps. Discrete black symbols: resonant frequencies of a cylindrical cavity $\left(R=10 \mathrm{c} / \omega_{\mathrm{p}}\right)$ with PEC walls filled by the gyrotropic material. The modes are labeled by the azimuthal quantum number $I$

Figure 2 shows the band structure of a magnetized plasma with $\omega_{\mathrm{c}}=0.8 \omega_{\mathrm{p}}$ (blue lines) for propagation in the xoy plane and transverse-magnetic (TM) polarization (the nontrivial field components are $E_{x}, E_{y}, H_{z}$ ). The medium has two photonic band-gaps. It is known that lossless electromagnetic continua may be topologically classified ${ }^{36}$, and in particular a lossless magnetized plasma $\left(\gamma=0^{+}\right)$has topologically nontrivial phases $^{18,44,45}$. The gap Chern numbers $\mathcal{C}_{\text {gap }, i}$ indicated in the insets of Fig. 2 are calculated as detailed in refs. ${ }^{36,38}$. They include the contributions of all bands below the band-gap, including the negative frequency bands ${ }^{38}$ (not shown in Fig. 2). The dispersion of the edge-states supported by a planar interface of the magnetized plasma (region $y>0$ ) and a PEC material (region $y<0$ ) is represented by the green lines in Fig. 2. Consistent with the bulk-edge correspondence principle, each band-gap supports a unidirectional edge-wave.

Consider now a cylindrical cavity filled with the magnetized plasma. The cavity lateral walls are PEC. Furthermore, to ease the analytical treatment the cavity cross-section is circular with radius $R$. In the Supplementary Note 4 , it is shown that the cavity modes have a magnetic field of the form $H_{z}=H_{0} I_{|l|}\left(\alpha_{\mathrm{ef}} \rho\right) \mathrm{e}^{i l \varphi}$, with $I_{|l|}$ the modified Bessel function of the 1st kind, $l=0, \pm 1, \ldots$ is the azimuthal quantum number, $\alpha_{\text {ef }}=\sqrt{-\varepsilon_{\text {ef }}} \omega / c$ and $\varepsilon_{\text {ef }}=$ $\left(\varepsilon_{\mathrm{t}}^{2}-\varepsilon_{\mathrm{g}}^{2}\right) / \varepsilon_{\mathrm{t}}$ is the effective permittivity of the gyrotropic material. The cavity modes satisfy the dispersion equation: $\alpha_{\mathrm{ef}} R \frac{I_{l l \mid}^{\prime}\left(\alpha_{\mathrm{ef}} R\right)}{I_{[l]}\left(\alpha_{\mathrm{ef}} R\right)}-l \frac{\varepsilon_{\mathrm{g}}}{\varepsilon_{\mathrm{t}}}=0$. The resonant frequencies for a cavity with radius $R=10 c / \omega_{\mathrm{p}}$ are represented in Fig. 2 as discrete black dots, in the spectral range determined by the band-gaps. As seen, the discrete dots follow closely the dispersion of the edge states associated with a planar interface (green lines), indicating that in the band-gaps the cavity modes are indeed localized near the lateral walls. This property is confirmed by Fig. 3a and 3b, which represent the profiles of two generic cavity modes in the 1st (lowfrequency) and 2nd (high-frequency) band-gaps, respectively. The modes of the 1st (2nd) gap have a positive (negative) azimuthal quantum number and circulate in the anti-clockwise (clockwise) direction.

The angular momentum spectral density was numerically calculated using the formula $\mathcal{L}_{\omega}=\mathcal{E}_{T, \omega} \sum_{\omega_{n}>0} \mathcal{L}^{(n)} \delta\left(\omega-\omega_{n}\right)$. An explicit expression for the normalized angular momentum of a generic mode $\left(\mathcal{L}^{(n)}\right)$ is given in the Supplementary Note 4 . The $\delta$ function is spread in the interval $\left(\omega_{n}+\omega_{n-1}\right) / 2<\omega<\left(\omega_{n+1}+\right.$ $\left.\omega_{n}\right) / 2$ as a rectangular pulse with height $2 /\left(\omega_{n+1}-\omega_{n-1}\right)$. Figure $4 \mathrm{a}$ and $\mathrm{b}$ depict the normalized spectral density $\tilde{\mathcal{L}}_{\omega} \equiv$ $\frac{\mathcal{L}_{\omega} c^{2} \pi}{\mathcal{E}_{T, \omega} A_{\text {tot }}}$ in the 1st and 2nd band-gaps, respectively, for cavities with radius $R=10 c / \omega_{\mathrm{p}}$ (dot-dashed green lines) and $R=20 c / \omega_{\mathrm{p}}$ (black lines). As seen, even though the cavity radius is only a few free-space wavelengths, the numerical results are consistent with the approximate identity (4): $\tilde{\mathcal{L}}_{\omega} \approx-\mathcal{C}_{\omega}=+1$ in the 1 st gap and $\tilde{\mathcal{L}}_{\omega} \approx-\mathcal{C}_{\omega}=-1$ in the 2 nd gap. In $R \rightarrow \infty$ limit the approximate identities become exact, and the angular momentum spectral density is exactly quantized and is determined by the gap Chern numbers: $\tilde{\mathcal{L}}_{\omega}=-\mathcal{C}_{\text {gap }, 1}$ in the 1st gap and $\tilde{\mathcal{L}}_{\omega}=-\mathcal{C}_{\text {gap }, 2}$ in the 2nd gap (dashed blue lines in Fig. 4).

Figure 5 represents the total angular momentum contribution of each band gap, defined as $\left\langle\mathcal{L}_{z, i}\right\rangle=\int_{\text {gap i }} \mathrm{d} \omega \mathcal{L}_{\omega}$, as a function of the temperature for $\omega_{\mathrm{p}} / 2 \pi=1 \mathrm{THz}$ and $\omega_{\mathrm{p}} / 2 \pi=10 \mathrm{THz}$. As could be anticipated, the system angular momentum due to the edge modes increases approximately linearly with the temperature because $\mathcal{E}_{T, \omega} \approx k_{\mathrm{B}} T$ for large $T$. The contributions of the band-gaps to the angular momentum have opposite signs. For very low-temperatures the contribution of the 2nd (highfrequency) gap dominates, and the total angular momentum due to edge waves is negative $\left(\left\langle\mathcal{L}_{z, 1}\right\rangle+\left\langle\mathcal{L}_{z, 2}\right\rangle<0\right)$. In the $T \rightarrow 0^{+}$ limit, the angular momentum is exclusively due to the quantum vacuum fluctuations. In this case, the zero-point energy (due to the gap edge-modes) flows in the clockwise direction around the lateral walls, i.e., in the direction determined by the electron "skipping orbits" (opposite to the bulk cyclotron orbits) ${ }^{18,46,47}$. In contrast, for moderately large temperatures the thermal-effects make $\left\langle\mathcal{L}_{z, 1}\right\rangle+\left\langle\mathcal{L}_{z, 2}\right\rangle$ positive and the contribution of the 1 st (low-frequency) gap dominates. It should be noted that bulk modes may also contribute to the fluctuation-induced angular momentum ${ }^{48,49}$, and hence $\left\langle\mathcal{L}_{z, 1}\right\rangle+\left\langle\mathcal{L}_{z, 2}\right\rangle$ only gives a partial (band-gap) component of $\left\langle\mathcal{L}_{z}\right\rangle$.

Outline of a microwave experiment. Next, we outline a possible microwave experiment to verify that the fluctuation-induced angular momentum density is nontrivial. Figure 6 depicts the metallic cavity filled with a topological material. A waveguide directional coupler is connected to the cavity through a multihole aperture (similar to a Bethe hole coupler $)^{50}$. In a microwave experiment (e.g., $\omega / 2 \pi \sim 10 \mathrm{GHz}$ ), the topological material can be implemented with a square array of ferrite rods biased with a static magnetic field, similar to ref. ${ }^{8}$. A small fraction of the thermal energy flowing near the cavity walls may be transferred to either port A or port B of the directional coupler. The directional coupler can be designed in such a manner that when the flow of thermal energy follows an anti-clockwise (clockwise) motion 
a

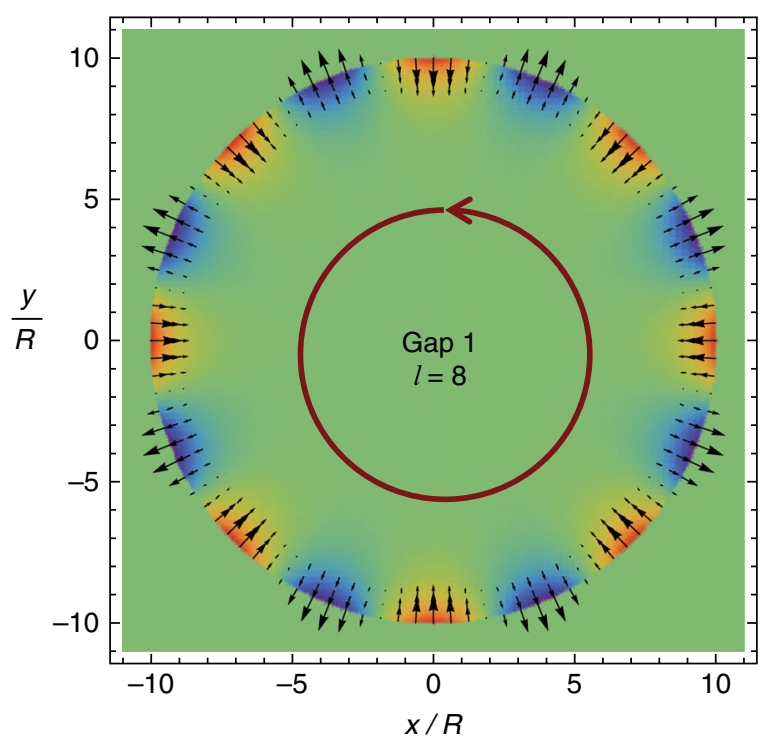

b
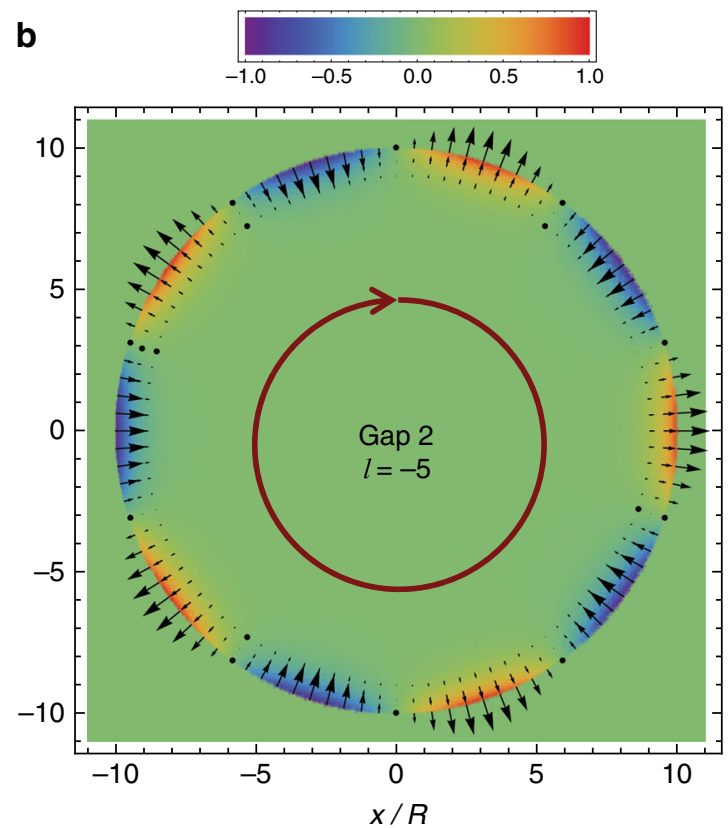

Fig. 3 Edge modes of the topological cavity. Each panel shows a density plot of a time snapshot of the magnetic field in a cylindrical cavity $\left(R=10 c / \omega_{\mathrm{p}}\right)$ for (a) the mode $I=8$ in the 1st (low-frequency) gap (propagating in the anti-clockwise direction) and (b) the mode $I=-5$ in the 2 nd (high-frequency) gap (propagating in the clockwise direction). The arrows represent the electric field lines

a

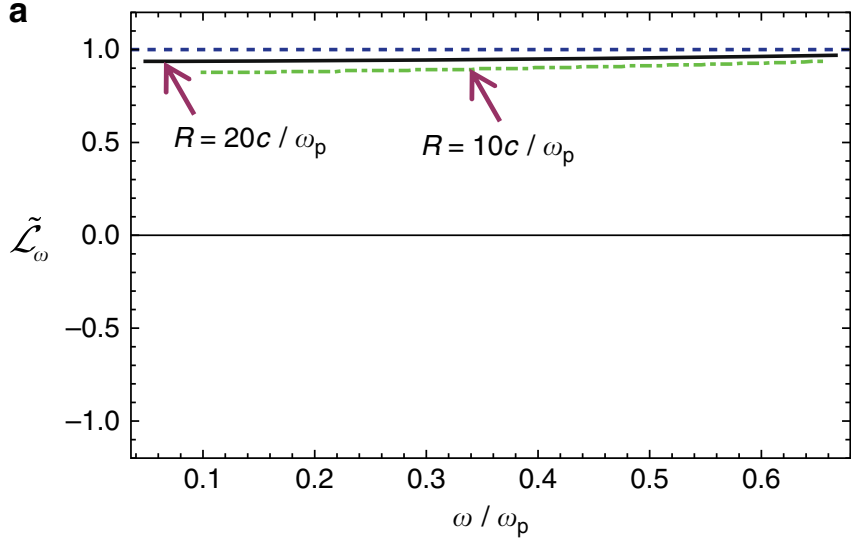

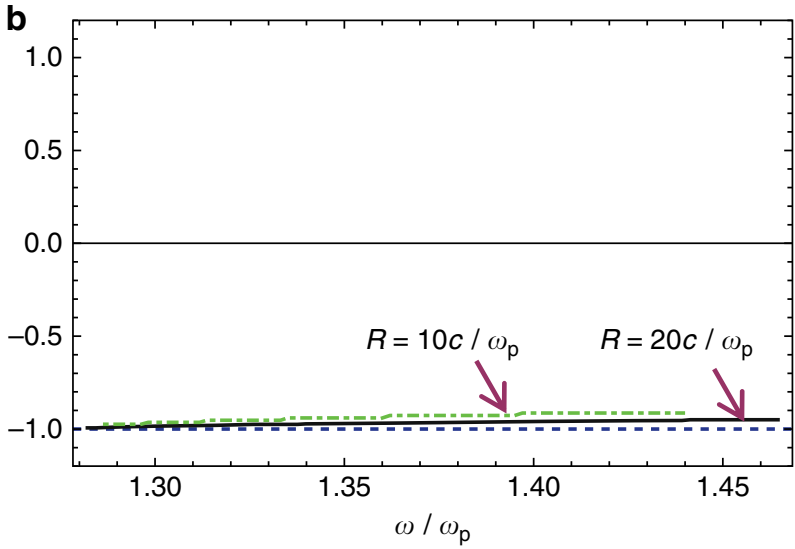

Fig. 4 Angular momentum spectral density as a function of frequency. The normalized angular momentum spectral density $\tilde{\mathcal{L}_{\omega}}$ is depicted for (a) the 1st band gap and (b) for the 2 nd band gap for a cylindrical cavity with radius $R=10 \mathrm{c} / \omega_{\mathrm{p}}$ (green dot-dashed line) and $R=20 \mathrm{c} / \omega_{\mathrm{p}}$ (black line). As $R \rightarrow \infty$, the angular momentum density becomes quantized (blue dashed horizontal line). The cyclotron frequency is $\omega_{\mathrm{c}}=0.8 \omega_{\mathrm{p}}$

(with respect to the $z$-axis) most of the energy is coupled to the port $\mathrm{B}$ (port A), and the other port is isolated ${ }^{50}$.

A measurement must perturb slightly the thermal equilibrium because otherwise the Poynting vector orbits are closed and the thermal flow is impossible to detect (in particular, the zero-point energy part of $\mathcal{E}_{T, \omega}$ cannot be directly measured) $)^{18}$. Hence, in an experiment the ports $\mathrm{A}$ and $\mathrm{B}$ must be held at a temperature $T_{0}$ different from the cavity temperature $T$. A detailed analysis reported in the Supplementary Note 5 shows that the difference between the power flows at ports $\mathrm{A}$ and $\mathrm{B}$ when the thermal equilibrium is perturbed $\left(T_{0}<T\right)$ is given by

$$
\delta p \equiv p_{\mathrm{B}}-p_{\mathrm{A}} \approx k_{\mathrm{B}}\left(T-T_{0}\right) \Delta C_{\mathrm{D}} \frac{\Delta \omega}{2 \pi},
$$

where $\Delta \omega$ is the bandwidth of the detecting circuit. The analysis assumes that both ports $\mathrm{A}$ and $\mathrm{B}$ are terminated by matched loads and that the material dissipation in the directional coupler is negligible due to the weak loss of metals at microwaves. The coefficient $\Delta C_{\mathrm{D}}$ is determined by the difference of the coupling strengths between ports A and B and all the cavity modes; it may be found with an independent calibration process, specifically from the $S$-parameters of the two-port network. Equation (6) is rather general and applies even when the frequency of interest is outside a band-gap and for any cavity size.

In the following, we concentrate on the case in which the detecting circuit bandwidth lies in a band-gap, so that $\Delta C_{\mathrm{D}}$ is entirely determined by the edge modes. Interestingly, because the power density transported by the edge modes is quantized and is independent of the system area (Supplementary Note 5), it follows that $\Delta C_{\mathrm{D}}$ will depend very little on the cavity cross- 

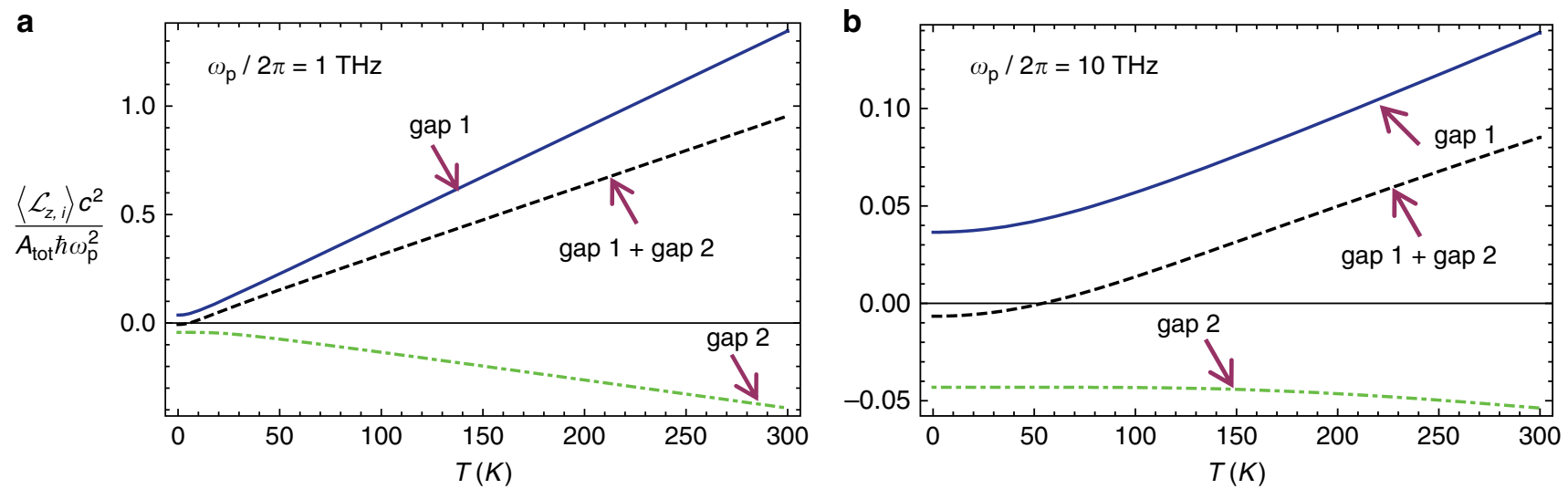

Fig. 5 Fluctuation induced angular momentum $\left\langle\mathcal{L}_{z, i}\right\rangle$ as a function of the temperature. The cylindrical cavity has radius $R>>c / \omega_{\mathrm{p}}$ and the plasma frequency is $(\mathbf{a}) \omega_{\mathrm{p}} / 2 \pi=1 \mathrm{THz}$ and $(\mathbf{b}) \omega_{\mathrm{p}} / 2 \pi=10 \mathrm{THz}$. The cyclotron frequency is $\omega_{\mathrm{c}}=0.8 \omega_{\mathrm{p}}$. Solid blue line: contribution from the $1 \mathrm{st}$ (low-frequency) band gap. Dot-dashed green line: contribution from the 2nd (high-frequency) band gap. Dashed black line: combined contribution of the two band gaps. Note that the contribution of the modes outside the spectral region of the band-gaps is not included in the calculation

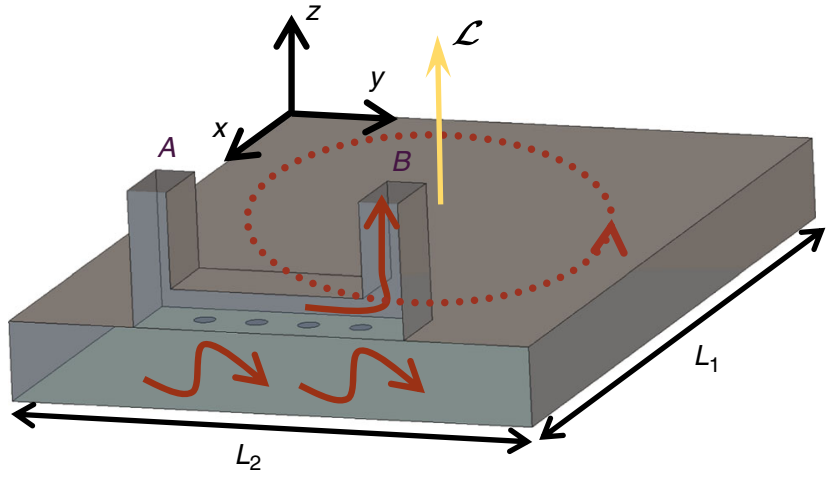

Fig. 6 Setup of a possible experiment at microwave frequencies. A metallic cavity is filled with a topological photonic crystal (not represented in the figure; e.g., a photonic crystal formed by ferrite rods ${ }^{8}$ ) and is connected to a waveguide coupler through multiple apertures on the top metallic plate. The directional coupler can be designed in such a manner that for an anticlockwise (clockwise) flow of thermal energy the coupled signal is transmitted mainly to port B (port A). The front wall of the cavity and the front wall of the directional coupler are not shown in order to visualize the interior of the structure

sectional area provided its diameter is a few times larger than the characteristic modal size so that the edge waves attached to opposite walls are well spatially separated. The typical modal size of the edge modes for a topological photonic crystal is on the order of the lattice constant ${ }^{8}$. Furthermore, it will also depend little on the specific location of the directional coupler in the cavity. Thus, a fingerprint of the topological gap is that $\delta p$ must be nearly independent of the cavity cross-section and of the location of the directional coupler. Note that outside a band-gap these properties do not hold.

For a reciprocal cavity its coupling with the two ports is symmetric and hence $\Delta C_{\mathrm{D}}$ vanishes and $\delta p=0$. Similarly, for a topologically trivial (but not necessarily reciprocal) gap $(\mathcal{C}=0)$ the number of edge states (if nonzero) circulating in counterclockwise direction is identical to the number of states circulating in the clockwise direction. This indicates that $\Delta C_{\mathrm{D}}$ is negligible when $\mathcal{C}=0$. Clearly, a significant imbalance between the net energy fluxes at ports $\mathrm{A}$ and $\mathrm{B}$ is only possible when $\mathcal{C} \neq 0$, and it will provide a clear signature of a nontrivial topology and confirm the circulation of thermal energy in closed orbits.

Ideally the directional coupler should ensure that the power transported by the modes circulating along a given direction is delivered to a single port. For example, if the coupler is designed in such a way that when the edge-modes propagate in an anticlockwise (clockwise) direction most of the thermal energy is coupled to port $\mathrm{B}(\mathrm{A})$ then $\Delta C_{\mathrm{D}}=-\operatorname{sgn}(\mathcal{C})\left|\Delta C_{\mathrm{D}}\right|$. Thus, the sign of $\delta p$ is linked to the sign of the Chern number. Furthermore, for an ideal coupler $\left|\Delta C_{\mathrm{D}}\right|$ represents the fraction of thermal energy rerouted from the cavity to the coupled port. Standard couplers can be very directive such that the ports coupling strength differs by four orders of magnitude ${ }^{50}$.

At ports $\mathrm{A}$ and $\mathrm{B}$ the thermal power may be collected by a microwave bolometer (radiometer) ${ }^{51,52}$. It is shown in the Supplementary Note 5, that the power rerouted from the topological cavity to the coupled port increases the noise temperature of the corresponding bolometer by $\delta T_{\mathrm{n}} \approx T\left|\Delta C_{\mathrm{D}}\right|$, where $T$ is the temperature of the cavity. For $\left|\Delta C_{\mathrm{D}}\right| \sim 0.1$ and $T=300 \mathrm{~K}$ the excess of noise temperature on the detector is $\delta T_{\mathrm{n}} \approx 30 \mathrm{~K}$. Thus, the bolometer needs to sufficiently sensitive to detect variations of the noise temperature on the order of some tens of Kelvin. Detectors cooled to a temperature on the order of $T_{0}=4 \mathrm{~K}$ can detect an excess of noise temperature as small as $\sim 1 \mathrm{~K}$ and are of widespread use in radio-astronomy ${ }^{53,54}$. Therefore, the proposed experiment seems to be within reach using cooled state-of-art bolometers with $T_{0} \leq \delta T_{\mathrm{n}}$.

\section{Discussion}

In summary, it was demonstrated that the fluctuation-induced angular momentum in a generic photonic insulator cavity with opaque-type boundaries has a quantized angular momentum in the photonic band-gaps. The quantized spectral-density depends on the net number of unidirectional edge-states at the cavity walls. For topological systems, the angular momentum is determined by the photonic Chern number, which thereby has a precise physical meaning as a "quantum" of the light-angular momentum spectral density. For $\mathbb{Z}_{2}$ topological photonic insulators with the time-reversal symmetry ${ }^{9}$ the spin-filtered $\mathcal{L}_{\text {" }} / A_{\text {tot }}$ is also quantized, but the contributions of the different "spins" cancel out. The nontrivial fluctuation-induced angular momentum may be experimentally verified by coupling the light that circulates around the cavity walls to a directional coupler and by 
detecting the imbalance between the energy sensed by the different arms of the coupler. We believe that the interpretation of the Chern number as a quantum of the angular momentum spectral density provides a deeper understanding about the intriguing role of topology in photonic systems ${ }^{40}$.

\section{Data availability}

The numerical data supporting the results of this article are available from the author upon request.

Received: 7 June 2018 Accepted: 21 December 2018

Published online: 21 January 2019

\section{References}

1. Haldane, F. D. M. Model for a Quantum Hall effect without Landau levels: condensed-matter realization of the "parity anomaly". Phys. Rev. Lett. 61, 2015-2018 (1988).

2. Kane, C. L. \& Mele, E. J. $Z_{2}$ topological order and the quantum spin Hall effect. Phys. Rev. Lett. 95, 146802 (2005).

3. Hasan, M. Z. \& Kane, C. L. Colloquium: topological insulators. Rev. Mod. Phys. 82, 3045 (2010).

4. Bernervig, B. A. \& Hughes, T., Topological Insulators and Topological Superconductors, (Princeton University Press, Princeton, New Jersey, 2013).

5. Haldane, F. D. M. Nobel lecture: topological quantum matter. Rev. Mod. Phys. 89, 040502 (2017).

6. Lu, L., Joannopoulos, J. D. \& Soljačić, M. Topological photonics. Nat. Photonics 8, 821-829 (2014).

7. Lu, L., Joannopoulos, J. D. \& Soljačić, M. Topological states in photonic systems. Nat. Phys. 12, 626-629 (2016).

8. Wang, Z., Chong, Y., Joannopoulos, J. D. \& Soljačić, M. Observation of unidirectional backscattering immune topological electromagnetic states. Nature 461, 772-775 (2009).

9. Khanikaev, A. B. et al. Photonic topological insulators. Nat. Mater. 12, 233-239 (2013).

10. Slobozhanyuk, A. et al. Three-dimensional all-dielectric photonic topological insulator. Nat. Photonics 11, 130-136 (2017).

11. Rechtsman, M. C. et al. Photonic Floquet topological insulators. Nature 496, 196-200 (2013).

12. He, C. et al. Photonic topological insulator with broken time-reversal symmetry. PNAS 113, 4924-4928 (2016).

13. Fleury, R., Khanikaev, A. \& Alù, A. Floquet topological insulators for sound. Nat. Commun. 7, 11744 (2016).

14. Haldane, F. D. M. \& Raghu, S. Possible realization of directional optical waveguides in photonic crystals with broken time-reversal symmetry. Phys. Rev. Lett. 100, 013904 (2008).

15. Raghu, S. \& Haldane, F. D. M. Analogs of quantum-Hall-effect edge states in photonic crystals. Phys. Rev. A 78, 033834 (2008).

16. Thouless, D. J., Kohmoto, M., Nightingale, M. P. \& den Nijs, M. Quantized Hall conductance in a two-dimensional periodic potential. Phys. Rev. Lett. 49, 405-408 (1982).

17. Landau, L. D. \& Lifshitz, E. M., Statistical Physics Part 2, Vol. 9 of Course on Theoretical Physics, Ch. VIII (Pergamon Press, Oxford, 1981).

18. Silveirinha, M. G. Topological angular momentum and radiative heat transport in closed orbits. Phys. Rev. B 95, 115103 (2017).

19. Leykam, D., Bliokh, K. Y., Huang, C., Chong, Y. D. \& Nori, F. Edge modes, degeneracies, and topological numbers in non-Hermitian systems. Phys. Rev. Lett. 118, 040401 (2017).

20. Shen, H., Zhen, B. \& Fu, L. Topological band theory for non-Hermitian Hamiltonians. Phys. Rev. Lett. 120, 146402 (2018).

21. Alvarez, V. M. M., Vargas, J. E. B., Berdakin M. \& Torres, L. E. F. F., Topological states of non-Hermitian systems, Eur. Phys. J. Spec. Top. https:// doi.org/10.1140/epjst/e2018-800091-5 (2018).

22. Yao, S. \& Wang, Z. Edge states and topological invariants of non-Hermitian systems. Phys. Rev. Lett. 121, 086803 (2018).

23. Kunst, F. K., Edvardsson, E., Budich, J. C. \& Bergholtz, E. J. Biorthogonal bulk-boundary correspondence in non-Hermitian systems. Phys. Rev. Lett. 121, 026808 (2018)

24. Silveirinha, M. G. $Z_{2}$ topological index for continuous photonic materials Phys. Rev. B 93, 075110 (2016)

25. Silveirinha, M. G. PTD symmetry protected scattering anomaly in optics. Phys. Rev. B 95, 035153 (2017).

26. Bliokh, K. Y., Bekshaev, A. Y. \& Nori, F. Extraordinary momentum and spin in evanescent waves. Nat. Commun. 5, 3300 (2014).
27. Bliokh, K. Y., Bekshaev, A. Y. \& Nori, F. Dual electromagnetism: helicity, spin, momentum, and angular momentum. New J. Phys. 15, 033026 (2013). Corrigendum: New J. Phys. 18, 089503 (2016).

28. Bliokh, K. Y., Bekshaev, A. Y. \& Nori, F. Optical momentum, spin, and angular momentum in dispersive media. Phys. Rev. Lett. 119, 073901 (2017),

29. Bliokh, K. Y., Bekshaev, A. Y. \& Nori, F. Optical momentum and angular momentum in dispersive media: From the Abraham-Minkowski debate to unusual properties of surface plasmon-polaritons. New J. Phys. 19, 123014 (2017).

30. Pfeifer, R. N. C., Nieminen, T. A., Heckenberg, N. R. \& Dunlop, H. R. Colloquium: momentum of an electromagnetic wave in dielectric media. Rev. Mod. Phys. 79, 1197 (2007).

31. Barnett, S. M. Resolution of the Abraham-Minkowski Dilemma. Phys. Rev. Lett. 104, 070401 (2010).

32. Silveirinha, M. G. Reexamination of the Abraham-Minkowski Dilemma. Phys Rev. A. 96, 033831 (2017).

33. Sakoda, K Optical Properties of Photonic Crystals. (Springer: Berlin, Heidelberg, New York, 2005.

34. Silveirinha, M. G. \& Maslovski, S. I. Exchange of momentum between moving matter induced by the zero-point fluctuations of the electromagnetic field. Phys. Rev. A. 86, 042118 (2012).

35. Zhu, L. \& Fan, S. Persistent directional current at equilibrium in nonreciprocal many-body near field electromagnetic heat transfer. Phys. Rev. Lett. 117, 134303 (2016)

36. Silveirinha, M. G. Chern invariants for continuous media. Phys. Rev. B 92, 125153 (2015)

37. Silveirinha, M. G. Bulk edge correspondence for topological photonic continua. Phys. Rev. B 94, 205105 (2016)

38. Silveirinha, M. G. Topological classification of Chern-type insulators by means of the photonic Green function. Phys. Rev. B 97, 115146 (2018).

39. Lannebère, S. A. \& Silveirinha, M. G. Link between the photonic and electronic topological phases in artificial graphene. Phys. Rev. B 97, 165128 (2018).

40. Silveirinha, M. G., Proof of the bulk-edge correspondence through a link between topological photonics and fluctuation-electrodynamics. Preprint at http://arxiv.org/abs/1804.02190 (2018).

41. Bittencourt, J. A. Fundamentals of Plasma Physics. 3rd edn (Springer-Verlag, New York, 2010)

42. Palik, E. et al. Coupled surface magnetoplasmon-optic phonon polariton modes on InSb. Phys. Rev. B 13, 2497 (1976).

43. Moncada-Villa, E., Hurtado, V. F., Garcia-Vidal, F. J., Garcia-Martin, A. \& Cuevas, J. C. Magnetic field control of near-field radiative heat transfer and the realization of highly tunable hyperbolic thermal emitters. Phys. Rev. B 92, 125418 (2015).

44. Gangaraj, S. A. H., Silveirinha, M. G. \& Hanson, G. W. Berry phase, Berry connection, and Chern number for a continuum bianisotropic material from a classical electromagnetics perspective. IEEE J. Multiscale Multiphys. Comput. Techn. 2, 3-17 (2017)

45. Gangaraj, S. A. H., Nemilentsau, A. \& Hanson, G. W. The effects of threedimensional defects on one-way surface plasmon propagation for photonic topological insulators comprised of continuum media. Sci. Rep. 6, 30055 (2016).

46. Halperin, B. I. Quantized Hall conductance, current-carrying edge states, and the existence of extended states in a two-dimensional disordered potential. Phys. Rev. B 25, 2185 (1982).

47. Darwin, C. G. The diamagnetism of the free electron. Math. Proc. Camb. Philos. Soc. 27, 86-90 (1931).

48. Thonhauser, T., Ceresoli, D., Vanderbilt, D. \& Resta, R. Orbital magnetization in periodic insulators. Phys. Rev. Lett. 95, 137205 (2005).

49. Ceresoli, D., Thonhauser, T., Vanderbilt, D. \& Resta, R. Orbital magnetization in crystalline solids: multi-band insulators, Chern insulators, and metals. Phys. Rev. B 74, 024408 (2006).

50. Pozar, D. M., Microwave Engineering, 3rd edn, Ch. 7 (Wiley, Hoboken, NJ, 2005).

51. Low, F. J. Low-temperature germanium bolometer. J. Opt. Soc. Am. 51, 1300-1304 (1961)

52. Richards, P. L. Bolometers for infrared and millimeter waves. J. Appl. Phys. 76, 1-24 (1994)

53. Penzias, A. A. \& Wilson, R. W. Measurement of the flux density of CAS A at $4080 \mathrm{Mc} / \mathrm{s}$. Astrophys. J. Lett. 142, 1149-1155 (1965).

54. Penzias, A. A. \& Wilson, R. W. A measurement of excess antenna temperature at $4080 \mathrm{Mc} / \mathrm{s}$. Astrophys. J. Lett. 142, 419-421 (1965).

\section{Acknowledgements}

This work is supported in part by Fundação para a Ciência e a Tecnologia grant number PTDC/EEI-TEL/4543/2014 and by Instituto de Telecomunicações under project UID/ $\mathrm{EEA} / 50008 / 2017$. 


\section{Author contribution}

M.G.S. conceived the research and carried out all the reported studies.

\section{Additional information}

Supplementary Information accompanies this paper at https://doi.org/10.1038/s41467018-08215-5

Competing interests: The author declares no competing interests.

Reprints and permission information is available online at http://npg.nature.com/ reprintsandpermissions/

Journal peer review information Nature Communications thanks the anonymous reviewers for their contribution to the peer review of this work.

Publisher's note: Springer Nature remains neutral with regard to jurisdictional claims in published maps and institutional affiliations. cc (i) Open Access This article is licensed under a Creative Commons Attribution 4.0 International License, which permits use, sharing, adaptation, distribution and reproduction in any medium or format, as long as you give appropriate credit to the original author(s) and the source, provide a link to the Creative Commons license, and indicate if changes were made. The images or other third party material in this article are included in the article's Creative Commons license, unless indicated otherwise in a credit line to the material. If material is not included in the article's Creative Commons license and your intended use is not permitted by statutory regulation or exceeds the permitted use, you will need to obtain permission directly from the copyright holder. To view a copy of this license, visit http://creativecommons.org/ licenses/by/4.0/.

(c) The Author(s) 2019 